\title{
YORP and Yarkovsky effects in asteroids (1685) Toro, (2100) Ra-Shalom, (3103) Eger, and (161989) Cacus
}

\author{
J. Ďurech ${ }^{1}$, D. Vokrouhlický ${ }^{1}$, P. Pravec ${ }^{2}$, J. Hanuš ${ }^{1}$, D. Farnocchia ${ }^{3}$, Yu. N. Krugly ${ }^{4}$, R. Y. Inasaridze ${ }^{5}$, \\ V. R. Ayvazian ${ }^{5}$, P. Fatka ${ }^{1,2}$, V. G. Chiorny ${ }^{4}$, N. Gaftonyuk ${ }^{4}$, A. Galád ${ }^{2}$, R. Groom ${ }^{6}$, K. Hornoch $^{2}$, H. Kučáková ${ }^{1,2}$, \\ P. Kušnirák ${ }^{2}$, M. Lehký ${ }^{1}$, O. I. Kvaratskhelia ${ }^{5}$, G. Masi ${ }^{7}$, I. E. Molotov ${ }^{8}$, J. Oey ${ }^{9}$, J. T. Pollock ${ }^{10}$, \\ V. G. Shevchenko ${ }^{4}$, J. Vrašstil ${ }^{1}$, and B. D. Warner ${ }^{11}$ \\ ${ }^{1}$ Institute of Astronomy, Faculty of Mathematics and Physics, Charles University, V Holešovičkách 2, 18000 Prague, \\ Czech Republic \\ e-mail: durech@sirrah.troja.mff.cuni.cz \\ 2 Astronomical Institute, Czech Academy of Sciences, Fričova 298, Ondřejov, Czech Republic \\ 3 Jet Propulsion Laboratory, California Institute of Technology, Pasadena, CA 91109, USA \\ 4 Institute of Astronomy of Kharkiv National University, Sumska Str. 35, 61022 Kharkiv, Ukraine \\ 5 Kharadze Abastumani Astrophysical Observatory, Ilia State University, K. Cholokoshvili Av. 3/5, 0162 Tbilisi, Georgia \\ ${ }^{6}$ Darling Range Observatory, Perth, WA, Australia \\ 7 Physics Department, University of Rome "Tor Vergata", via della Ricerca Scientifica 1, 00133 Rome, Italy \\ 8 Keldysh Institute of Applied Mathematics, RAS, Miusskaya 4, 125047 Moscow, Russia \\ 9 Blue Mountains Observatory, 94 Rawson Pde. Leura, NSW 2780, Australia \\ 10 Physics and Astronomy Department, Appalachian State University, 525 Rivers St., Boone, NC 28608, USA \\ 11 Center for Solar System Studies - Palmer Divide Station, 446 Sycamore Ave., Eaton, CO 80615, USA
}

Received 29 June 2017 / Accepted 20 September 2017

\section{ABSTRACT}

\begin{abstract}
Context. The rotation states of small asteroids are affected by a net torque arising from an anisotropic sunlight reflection and thermal radiation from the asteroids' surfaces. On long timescales, this so-called YORP effect can change asteroid spin directions and their rotation periods.

Aims. We analyzed lightcurves of four selected near-Earth asteroids with the aim of detecting secular changes in their rotation rates that are caused by YORP or at least of putting upper limits on such changes.

Methods. We use the lightcurve inversion method to model the observed lightcurves and include the change in the rotation rate $\mathrm{d} \omega / \mathrm{d} t$ as a free parameter of optimization. To enlarge the time line of observations and to increase the sensitivity of the method, we collected more than 70 new lightcurves. For asteroids Toro and Cacus, we used thermal infrared data from the WISE spacecraft and estimated their size and thermal inertia by means of a thermophysical model. We also used the currently available optical and radar astrometry of Toro, Ra-Shalom, and Cacus to infer the Yarkovsky effect.

Results. We detected a YORP acceleration of $\mathrm{d} \omega / \mathrm{d} t=(1.9 \pm 0.3) \times 10^{-8} \mathrm{rad} \mathrm{d}^{-2}$ for asteroid Cacus. The current astrometric data set is not sufficient to provide detection of the Yarkovsky effect in this case. For Toro, we have a tentative $(2 \sigma)$ detection of YORP from a significant improvement of the lightcurve fit for a nonzero value of $\mathrm{d} \omega / \mathrm{d} t=3.0 \times 10^{-9} \mathrm{rad} \mathrm{d}^{-2}$. We note an excellent agreement between the observed secular change of the semimajor axis $\mathrm{d} a / \mathrm{d} t$ and the theoretical expectation for densities in the $2-2.5 \mathrm{~g} \mathrm{~cm}^{-3}$ range. For asteroid Eger, we confirmed the previously published YORP detection with more data and updated the YORP value to $(1.1 \pm 0.5) \times 10^{-8} \mathrm{rad} \mathrm{d}^{-2}$. We also updated the shape model of asteroid Ra-Shalom and put an upper limit for the change of the rotation rate to $|\mathrm{d} \omega / \mathrm{d} t| \lesssim 1.5 \times 10^{-8} \mathrm{rad} \mathrm{d}^{-2}$. Ra-Shalom has a greater than $3 \sigma$ Yarkovsky detection with a theoretical value consistent with observations assuming its size and/or density is slightly larger than the nominally expected values. Using the convex shape models and spin parameters reconstructed from lightcurves, we computed theoretical YORP values and compared them with those measured. They agree with each other within the expected uncertainties of the model.
\end{abstract}

Key words. minor planets, asteroids: general - radiation mechanisms: thermal - techniques: photometric

\section{Introduction}

The rotation state of small $(\$ 30 \mathrm{~km})$ asteroids can be affected on long timescales by a net torque that is caused by directly scattered sunlight and thermal radiation from the surfaces of the asteroids. This so-called YORP effect can change the directions of the rotation axis and the rotation rates (Bottke et al. 2006; Vokrouhlický et al. 2015) and has direct consequences for the distribution of asteroid rotation periods (Pravec et al. 2008) and obliquities (Hanuš et al. 2013; Pravec et al. 2012b). The YORP effect is also believed to be the driving mechanism for creating asteroid binaries and pairs by rotation fission (Pravec et al. 2010; Margot et al. 2015).

In a similar vein, orbits of small asteroids are affected by the Yarkovsky effect, a net reaction force from the thermal radiation of a rotating body with nonzero thermal inertia; it has crucial consequences for the evolution of the main asteroid belt, for the supply of the near-Earth asteroid (NEA) population, and for impact hazard assessment (see the review by Vokrouhlický et al. 2015). The Yarkovsky drift has been detected for more than 
one hundred NEAs (e.g., Farnocchia et al. 2013, Greenberg et al. 2017).

While the evolution of the spin axis cannot be detected from current photometric data, a change in the rotation rate can be detected because time-resolved photometry is very sensitive to even a small secular change in the rotation period. So far, YORP-driven acceleration of the rotation period has been directly detected in five asteroids (see the review by Vokrouhlický et al. 2015) and there are indirect detections of YORP-driven evolution of spins of members of asteroid families (Vokrouhlický et al. 2003; Carruba et al. 2016; Paolicchi \& Knežević 2016). Additional direct detections are needed if we want to compare real values of YORP with those predicted by theoretical models (Rozitis \& Green 2013; Golubov et al. 2014; Lowry et al. 2014; Ševeček et al. 2015, for example). The YORP-driven evolution of asteroid rotation plays a crucial role in the dynamical evolution of the whole asteroid population and only new measurements of the YORP effect together with theoretical models will enable us to create a selfconsistent model of this process.

To enlarge the sample of asteroids with a YORP detection, we analyzed archival lightcurves and new data of four NEAs that, according to the estimated YORP magnitude, should have a detectable deviation from the constant-period rotation. We also reevaluate observation constraints for the Yarkovsky effect and put them into context with the size and thermal inertia we derived for our targets.

\section{YORP detection through lightcurve inversion}

To look for possible secular changes in the rotation period, we used the lightcurve inversion method of Kaasalainen et al. (2001). Kaasalainen et al. (2003) slightly modified the method so that it included one more free parameter in the optimization: the change of the rotation rate $v=\mathrm{d} \omega / \mathrm{d} t$. We applied this modified lightcurve inversion to archived photometric lightcurves (references given below) and our new observations (Tables 1, A.1-A.3). For each asteroid, we reconstructed its convex shape model and tested whether a nonzero $v$ value provides a significantly better fit to the data than a model with constant period $(v=0)$. We estimated the uncertainties of the derived parameters with the same approach as Durech et al. (2012) from the $\chi^{2}$ distribution with a given degrees of freedom. If not stated otherwise, the reported uncertainties are $1 \sigma$.

\section{1. (3103) Eger}

This is one of five asteroids in which YORP has been detected. Durech et al. (2012) determined the YORP acceleration to $v=$ $(1.4 \pm 0.6) \times 10^{-8} \mathrm{rad} \mathrm{d}^{-2}(3 \sigma$ error $)$, and included a "warning" that data from upcoming apparitions would be needed to confirm this detection. By adding more observations from 2014 and 2016 (Warner 2017) and our two lightcurves from 2017 (see Table 1), we confirmed previous results and derived an updated value $v=(1.1 \pm 0.5) \times 10^{-8} \mathrm{rad} \mathrm{d}^{-2}(3 \sigma$ error $)$ with slightly better precision. For a realistic estimate of the uncertainty interval, we used the same approach as Vokrouhlický et al. $(2011,2017)$ by assuming that the $3 \sigma$ uncertainty interval is defined by all solutions with $\chi^{2}<(1+3 \sqrt{2 v}) \chi_{\min }^{2}$, where $\chi_{\min }^{2}$ was the $\chi^{2}$ of the best model and $v$ was the number of degrees of freedom $(v \sim 5500$ in case of Eger). The dependence of $\chi^{2}$ on the YORP parameter $v$ is shown in Fig. 1 for $v$ between 0 and $2.0 \times 10^{-8} \mathrm{rad} \mathrm{d}^{-2}$. The $3 \sigma$ uncertainty corresponds to an increase in $\chi^{2}$ of about $6 \%$.

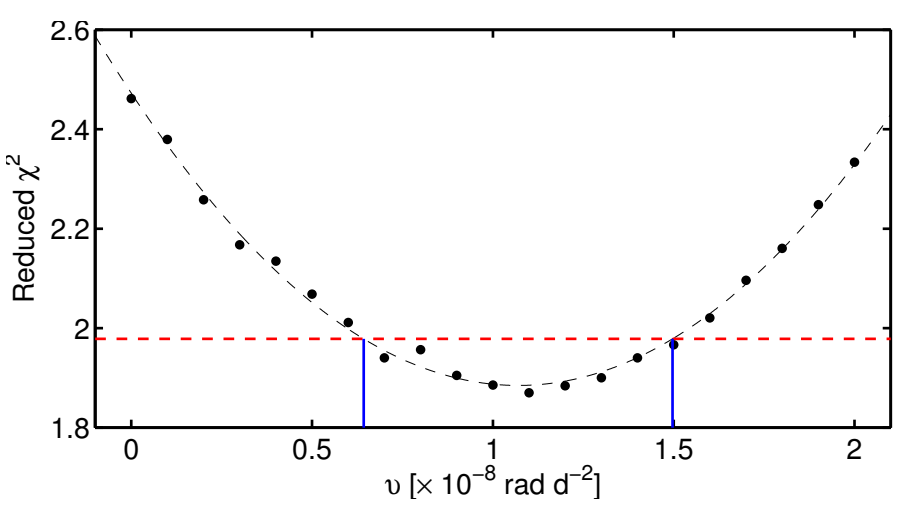

Fig. 1. Dependence of the goodness of the fit measured by the reduced $\chi^{2}$ on the YORP parameter $v$ for asteroid Eger. The best fit is for $v=1.1 \times 10^{-8} \mathrm{rad} \mathrm{d}^{-2}$. The dashed curve is a quadratic fit of the data points. The dashed red line indicates a $6 \%$ increase in the $\chi^{2}$, which defines our $3 \sigma$ uncertainty interval given the number of degrees of freedom.

Table 1. Aspect data for new observations of (3103) Eger.

\begin{tabular}{ccccccc}
\hline \hline Date & $\begin{array}{c}r \\
{[\mathrm{AU}]}\end{array}$ & $\begin{array}{c}\Delta \\
{[\mathrm{AU}]}\end{array}$ & $\begin{array}{c}\alpha \\
{[\mathrm{deg}]}\end{array}$ & $\begin{array}{c}\lambda \\
{[\mathrm{deg}]}\end{array}$ & $\begin{array}{c}\beta \\
{[\mathrm{deg}]}\end{array}$ & Obs. \\
\hline 20170205.8 & 1.487 & 0.523 & 13.6 & 152.8 & 13.6 & Ond \\
20170216.1 & 1.534 & 0.564 & 11.4 & 146.3 & 17.8 & Ond \\
\hline
\end{tabular}

Notes. The table lists Eger's distance from the Sun $r$ and from the Earth $\Delta$, the solar phase angle $\alpha$, the geocentric ecliptic coordinates of the asteroid $(\lambda, \beta)$, and the observatory (Ond - Ondřejov, $65 \mathrm{~cm}$ ).

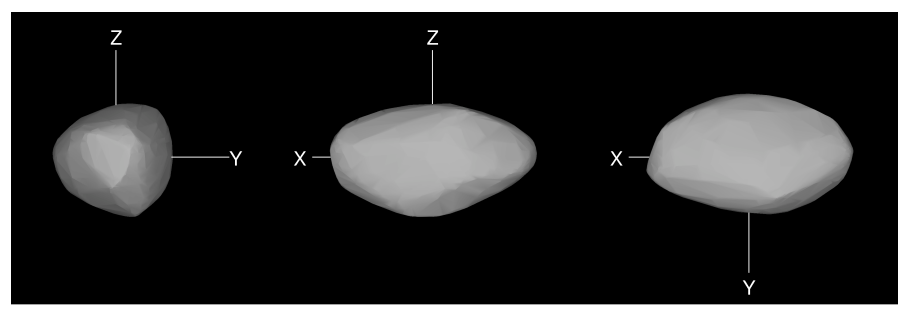

Fig. 2. Shape model of (1685) Toro shown from equatorial level (left and center, $90^{\circ}$ apart) and pole-on (right).

The new convex shape model is very similar to that published by Durech et al. (2012). However, we recall that to significantly decrease the uncertainty of the $v$ value, it is necessary to extend the data arc with observations from the next apparitions.

\section{2. (1685) Toro}

For Toro we used archived lightcurves from 1972 (Dunlap et al. 1973), 1998 (Hoffmann \& Geyer 1990), 2007 (Higgins 2008), 2008 (Higgins et al. 2008), 2010 (Higgins 2011; Oey 2011), and 2013 (Warner 2013), and we also observed new lightcurves (see Table A.1). From this data set, we reconstructed the shape model (Fig. 2), the rotation period $P=(10.19782 \pm 0.00003) \mathrm{h}$ for 8.5 July 1972 (the date of the first photometric observation), the pole direction $(\lambda, \beta)=\left(71 \pm 10^{\circ},-69 \pm 5^{\circ}\right)$ (corresponding to obliquity $\left.\epsilon=161 \pm 6^{\circ}\right)$, and $v=3.0 \times 10^{-9} \mathrm{rad} \mathrm{d}^{-2}$. For this value of $v$, the $\chi^{2}$ drops by $11 \%$ with respect to $\chi^{2}$ for $v=0$. The formal phase shift over the interval of 44 years corresponding to this value of $v$ is only $22^{\circ}$. The difference between 
$1972 / 7 / 9.4$

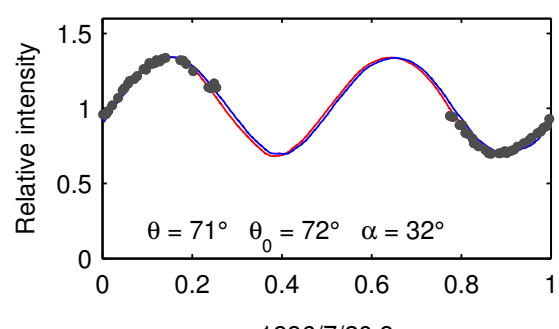

$1996 / 7 / 20.9$

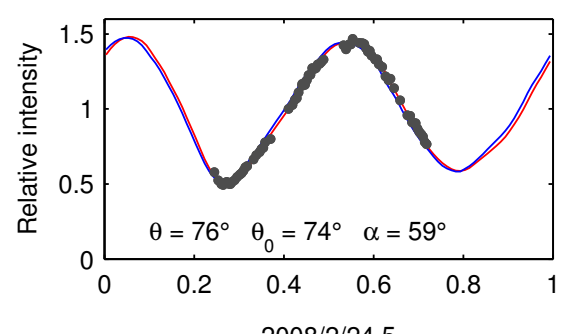

$2008 / 2 / 24.5$

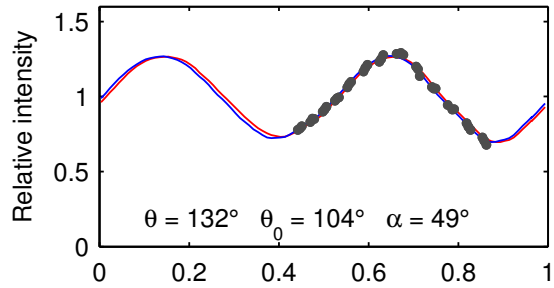

$2013 / 4 / 8.2$

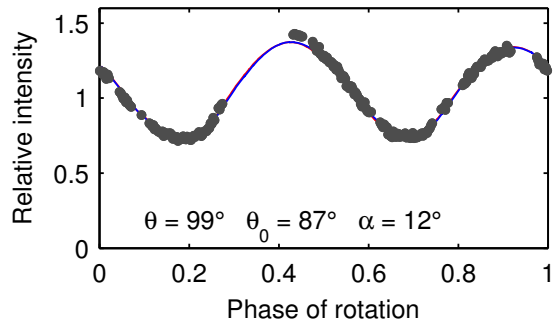

$1972 / 8 / 3.5$

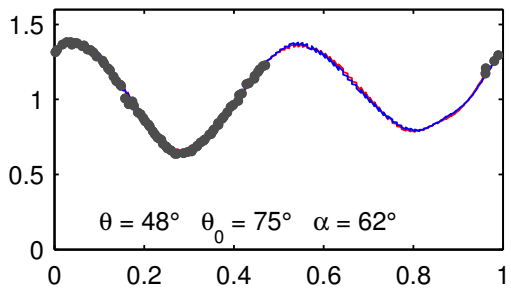

$2004 / 7 / 24.0$

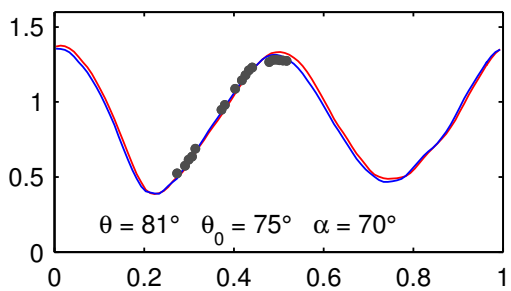

$2010 / 5 / 9.6$

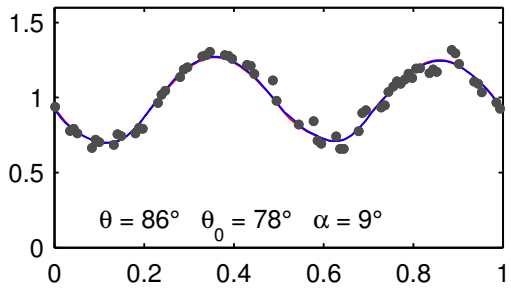

$2015 / 7 / 1.5$

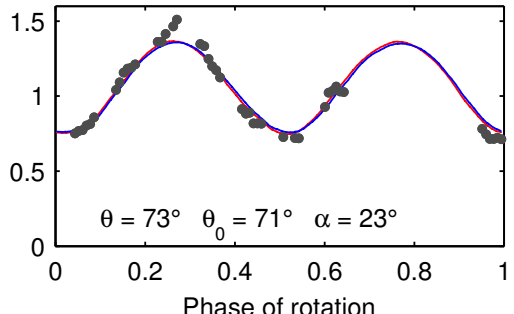

$1988 / 8 / 6.0$

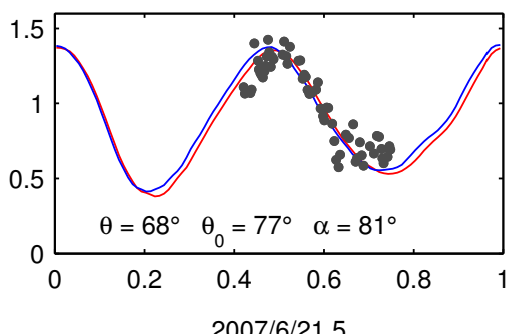

$2007 / 6 / 21.5$

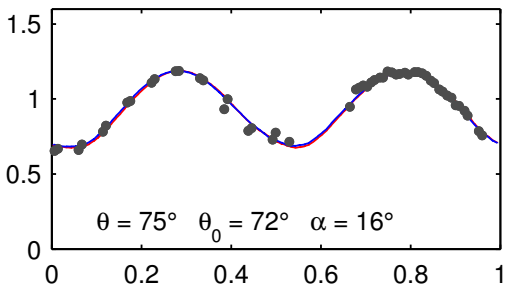

$2012 / 7 / 9.9$

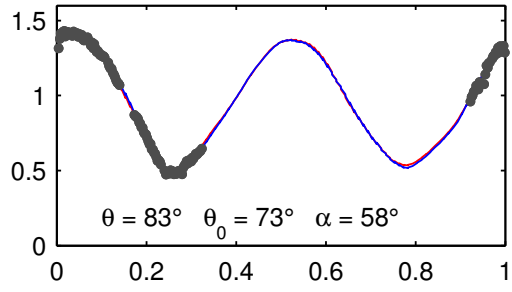

$2016 / 2 / 29.8$

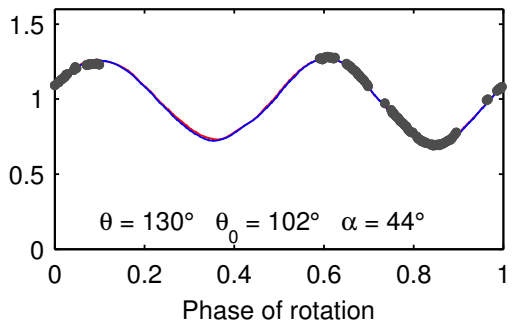

Fig. 3. Example lightcurves of (1685) Toro shown with the synthetic lightcurves produced by the best-fit constant-period model (blue) and with YORP (red). The geometry is described by the aspect angle $\theta$, the solar aspect angle $\theta_{0}$, and the solar phase angle $\alpha$.

the constant period and YORP model is most pronounced for lightcurves from 1996. They are also crucial for YORP detection. When the four lightcurves observed in 1996 are removed, the difference between the YORP model with the best-fit value $v=2.3 \times 10^{-9} \mathrm{rad} \mathrm{d}^{-2}$ and a constant period model with $v=0$ is only $4 \%$ in $\chi^{2}$. To confirm this tentative YORP detection, additional data from future apparitions are needed. The fit to the selected lightcurves is shown in Fig. 3.

To better characterize this asteroid, we also used observations of the Wide-field Infrared Survey Explorer (WISE) satellite (Wright et al. 2010; Mainzer et al. 2011). WISE observed Toro in two epochs (10 February and 15 July) in 2010, the data from the W3 $(11 \mu \mathrm{m})$ and W4 $(23 \mu \mathrm{m})$ filters are available through the IRSA/IPAC archive. We checked the data against the quality and reliability criteria described in Alí-Lagoa et al. (2016). Using our shape model and spin parameters, we applied the thermophysical model of Lagerros $(1996,1997,1998)$ to derive the thermophysical properties. The best fit with the reduced $\chi^{2}=1.4$ is for thermal inertia $260_{-110}^{+140} \mathrm{~J} \mathrm{~m}^{-2} \mathrm{~s}^{-0.5} \mathrm{~K}^{-1}$, high roughness, and albedo $0.13 \pm 0.03$, assuming the values $H=13.9 \mathrm{mag}$ and $G=0.11$ from the database of asteroid absolute magnitudes and slopes (Muinonen et al. 2010; Oszkiewicz et al. 2011). The size of the asteroid is $3.5_{-0.4}^{+0.3} \mathrm{~km}$, which is in good agreement with the mean effective diameter of $\sim 3.3 \mathrm{~km}$ derived by Ostro et al. (1983) from radar observations. The fit to the data is shown in Fig. 4.

\section{3. (161989) Cacus}

The first lightcurves of Cacus come from 1978 observations of Schuster et al. (1979) and Degewij et al. (1978). During 2003, the asteroid was observed from Ondřejov observatory; Koehn et al. (2014) observed one lightcurve in 2009; and we observed this asteroid in 2014-16 at La Silla. The whole set covers 20 years and five apparitions (see Table A.2). On 17 February 2015 we measured the color index in the Johnson-Cousins photometric system $(V-R)=(0.486 \pm 0.015)$ mag. From observations taken on 8 and 15 December 2015, we derived the mean absolute magnitude $H=(17.51 \pm 0.19)$ mag assuming the phase slope parameter $G=0.24 \pm 0.11$, which is the mean $G$ value for S- and Q-type asteroids (Pravec et al. 2012a).

We applied the lightcurve inversion to the photometric data set and derived a unique shape model. The fit to lightcurves with $v=0$ was not satisfactory, but if we allowed the rotation rate to change, we got a significantly better fit (see Fig. 5). Our final model has the pole direction $\lambda=(254 \pm 5)^{\circ}, \beta=(-62 \pm 2)^{\circ}$ (corresponding obliquity is $\epsilon=178 \pm 3^{\circ}$ ) and rotation period 
$P=(3.755067 \pm 0.000002) \mathrm{h}$ for 28.5 February 1978 . The best value for the change in the rotation rate is $v=(1.9 \pm 0.3) \times$ $10^{-8} \mathrm{rad} \mathrm{d}^{-2}$. The shape model is shown in Fig. 6 .

In Fig. 7, we show the phase shift between the best constantperiod model $(P=3.755054 \mathrm{~h})$ and the real data. For each observed lightcurve, we created a corresponding smooth synthetic lightcurve produced by the best-fit constant-period model and then computed the phase shift of synthetic data that produced the best match between the two lightcurves. These values are shown in the plot together with the error bars estimated from the number of points and the level of noise in each lightcurve. If YORP changes the rotation rate, this $\mathrm{O}-\mathrm{C}$ difference should be a quadratic function of time. The trend is not very clear mainly because of three lightcurves from 2003, but they have large error bars and a small number of points, so their contribution is less significant. The formal YORP coefficient obtained by fitting a second-order polynomial to the phase-shift points in Fig. 7 is $1.0 \times 10^{-8} \mathrm{rad} \mathrm{d}^{-2}$.

The detection of the YORP acceleration is critically dependent on the first two lightcurves from 1978. If we exclude them from the data set, the observations span only 13 years and the difference between the constant period and YORP model is not statistically significant: both models provide essentially the same fit to the data and the phase offset between the models for $v=1.9 \times 10^{-8} \mathrm{rad} \mathrm{d}^{-2}$ is only $\sim 3^{\circ}$. However, because the two lightcurves were obtained by independent observers and instruments (Degewij et al. 1978; Schuster et al. 1979), it is not likely that they were both shifted in time the same way to mimic the YORP effect.

Similarly to Toro, we also used observations of the WISE spacecraft, which observed Cacus in 2010. Using our shape model and spin parameters, we applied the thermophysical model and derived the thermophysical properties. Because the thermal data in W4 filter have large uncertainties, they only provide loose constraints to the thermal inertia and the size of the asteroid. The best fit with the reduced $\chi^{2}=0.7$ is for a thermal inertia around $500-800 \mathrm{~J} \mathrm{~m}^{-2} \mathrm{~s}^{-0.5} \mathrm{~K}^{-1}$, but all of the values in the range $250-2000 \mathrm{~J} \mathrm{~m}^{-2} \mathrm{~s}^{-0.5} \mathrm{~K}^{-1}$ with low to medium surface roughness provide a very good fit to the data (Fig. 8). The modeled size of Cacus is $(1.0 \pm 0.2) \mathrm{km}$, which gives the albedo $p_{V}=0.18 \pm 0.08$.

\section{4. (2100) Ra-Shalom}

The modeling of Ra-Shalom aiming at YORP detection was done by Durech et al. (2012) who used the data set from 1978 to 2009. We extended the set with lightcurves from two additional apparitions in 2013 and 2016 (see Table A.3). On 8 October 2016 we measured the color index in the Johnson-Cousins photometric system $(V-R)=(0.398 \pm 0.010)$ mag. There is still no detectable signal of a YORP torque; the YORP model provides virtually the same $\chi^{2}$ as the constant period model. However, with the enlarged time line, we were able to reduce the $3 \sigma$ uncertainty interval of YORP to $-1.0 \times 10^{-8}<v<1.5 \times 10^{-8} \mathrm{rad} \mathrm{d}^{-2}$, which is about 2-3 times tighter than in Durech et al. (2012). Because the data set is dominated by the more recent lightcurves, the discrepancy between the data and the model are most pronounced for the 1978 and 1981 lightcurves. We also updated the spin pole orientation and decreased its uncertainty (compared to the rather large uncertainty of pole direction in Durech et al. 2012): $\lambda=(292 \pm 15)^{\circ}, \beta=(-65 \pm 10)^{\circ}, P=(19.8200 \pm 0.0003) \mathrm{h}$, $\epsilon=166 \pm 12^{\circ}$.
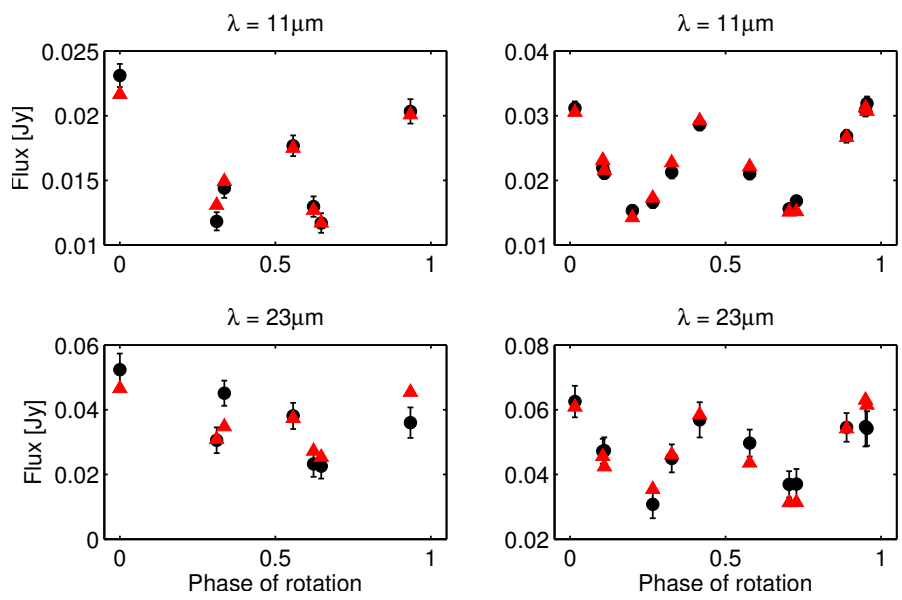

Fig. 4. Comparison between the model (red triangles) and Toro thermal infrared data observed by WISE on 10 February 2010 (left), and 15 July 2010 (right).

\section{Comparison with the theoretical model}

Here we estimate how the detected change in rotation rate for (161989) Cacus, its tentative value for (1685) Toro, and limits set in the (2100) Ra-Shalom case agree with the theoretical expectations from the YORP effect. The analysis of (3103) Eger was already presented by Durech et al. (2012). We also reevaluate the observational constraints of the Yarkovsky effect for these asteroids and compare them with our model. This is not a straightforward task. First, it requires a thermophysical model of the analyzed asteroid, which depends on a number of poorly known parameters. Second, it has been recognized that the YORP strength is sensitive to small-scale irregularities of the asteroid shape, which are far beyond the resolution of our coarse convex models (e.g., Vokrouhlický et al. 2015, and references therein). In this situation we decided to adopt the simplest possible model and leave the door open for further improvements in the future. As a consequence, the real uncertainties of our theoretical predictions are larger than the formal ones corresponding to the uncertainty of the input parameters.

In particular, we use the one-dimensional heat diffusion model of Čapek \& Vokrouhlický $(2004 ; 2005)$. This approach is able to treat the self-shadowing of surface facets. However, this capability is not implemented in our computation where we only use a coarse convex model from lightcurve inversion techniques. We treat each facet independently and the time-dependent heat diffusion propagates to the depth below the surface. We assumed that the core is isothermal and treat the surface boundary condition in its nonlinear form (e.g., Čapek \& Vokrouhlický 2004, 2005). The formulation requires setting the values of the surface thermal conductivity $K$, density $\rho_{\mathrm{s}}$, and heat capacity $C$. These quantities are also traditionally combined into the surface thermal inertia $\Gamma=\sqrt{K \rho_{\mathrm{s}} C}$. If the surface is known down to small scales (centimeters to decimeters), there are more complications to affect the YORP strength: (i) shadowing and mutual thermal irradiation of the surface facets (e.g., Rozitis \& Green 2012, 2013) and (ii) thermal communication of the surface facets (e.g., Golubov \& Krugly 2012; Ševeček et al. 2016). While these effects have limited influence on the accuracy of the global thermal acceleration (the Yarkovsky effect), they can significantly change the global thermal torque (the YORP effect). Overall, the self-irradiation tends to decrease the magnitude of the YORP effect (e.g., Rozitis \& Green 2013), while the thermal communication of the surface facets introduces a systematic trend that 
$1978 / 3 / 1.2$

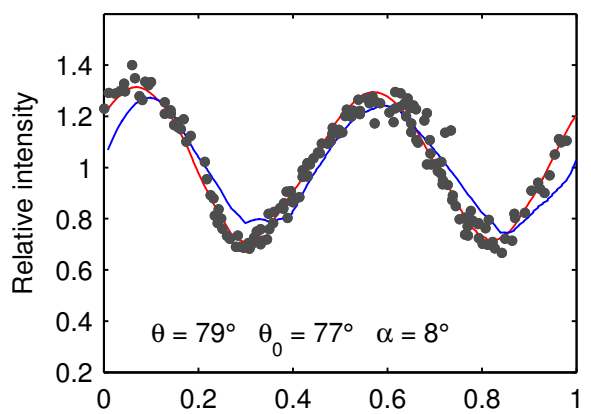

$2014 / 12 / 21.3$

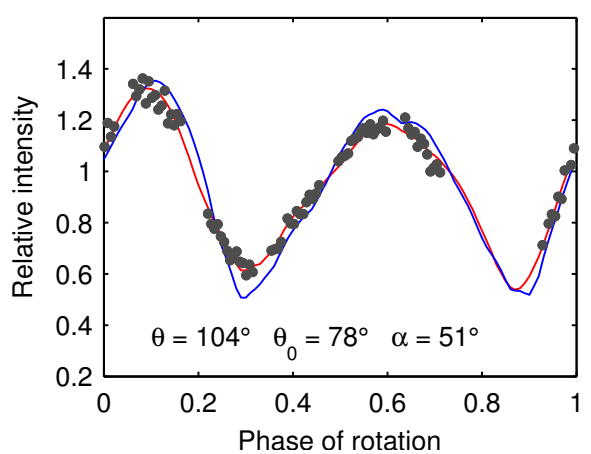

$2003 / 2 / 18.1$

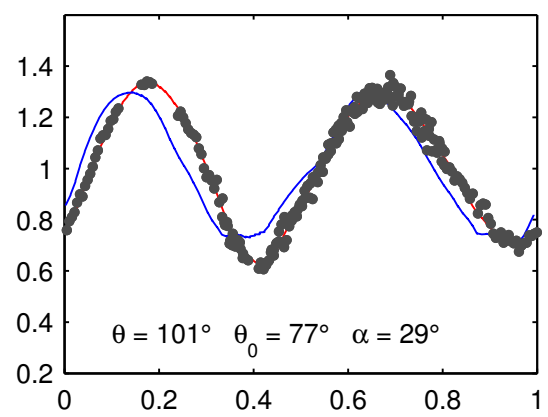

$2015 / 11 / 5.3$

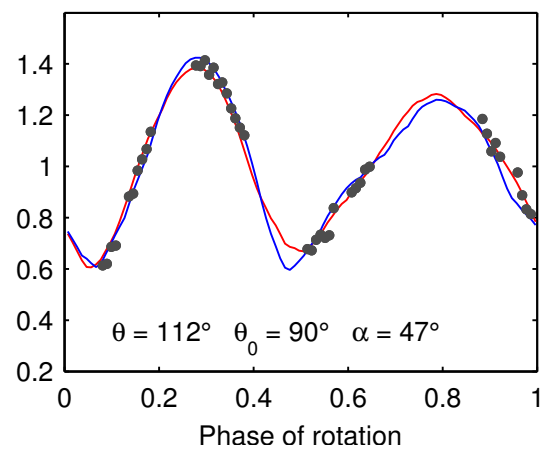

$2009 / 2 / 19.4$

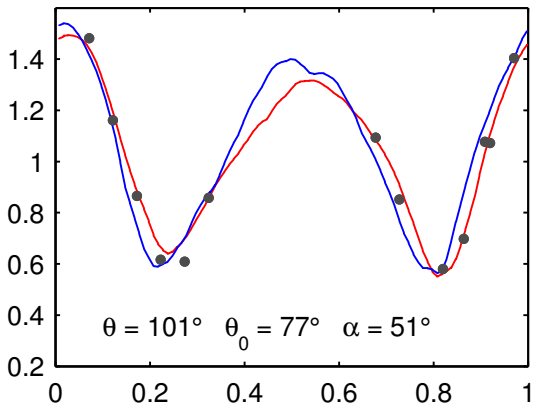

$2016 / 10 / 6.1$

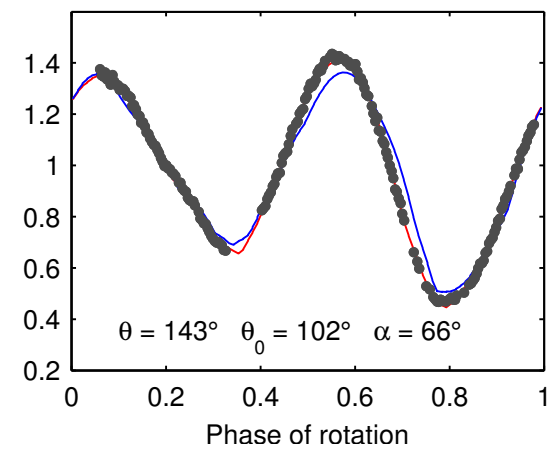

Fig. 5. Example lightcurves of (161989) Cacus shown with the synthetic lightcurves produced by the best-fit constant-period model (blue) and with YORP (red). The geometry is described by the aspect angle $\theta$, the solar aspect angle $\theta_{0}$, and the solar phase angle $\alpha$.

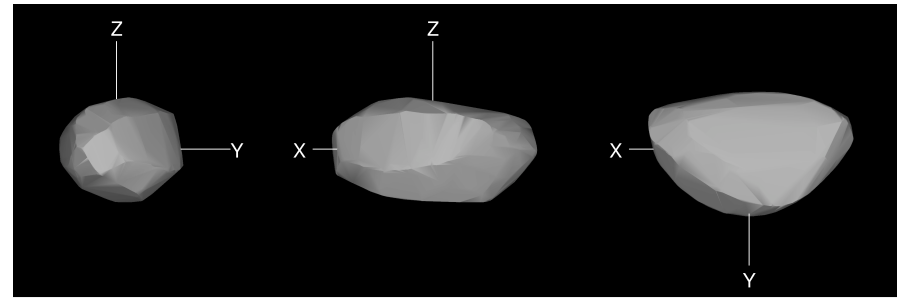

Fig. 6. Shape model of (161989) Cacus shown from equatorial level (left and center, $90^{\circ}$ apart) and pole-on (right).

accelerates the rotation instead of decreasing it, as caused by the YORP effect (e.g., Golubov \& Krugly 2012). So the typical mismatch arising from the simplified approach using a smooth, convex shape model is overprediction of the YORP strength, and apparent symmetry in acceleration and deceleration of the rotation rate (e.g., Čapek \& Vokrouhlický 2004). Because the thermal effects take place only in the thin surface layer (typically $\leq 1 \mathrm{~m}$ ), the isothermal core bulk density $\rho_{\mathrm{b}}$ is the next parameter that needs to be specified. Finally, our model requires an equivalent size $D$ of the asteroid (i.e., diameter of a sphere with the same volume as the asteroid), the orientation of the spin axis, and the rotation period. The last two are taken from our solution in Sect. 2, and the size is estimated from infrared observations or radar data.

Once the thermal model converges to the solution of the surface temperature distribution at any time during the revolution about the Sun, we can use the converged solution to compute both thermal force and torque. By numerically averaging over one revolution about the Sun, we derive estimates of the Yarkovsky and YORP effects. The former is represented with a single parameter, namely the secular change in semimajor axis

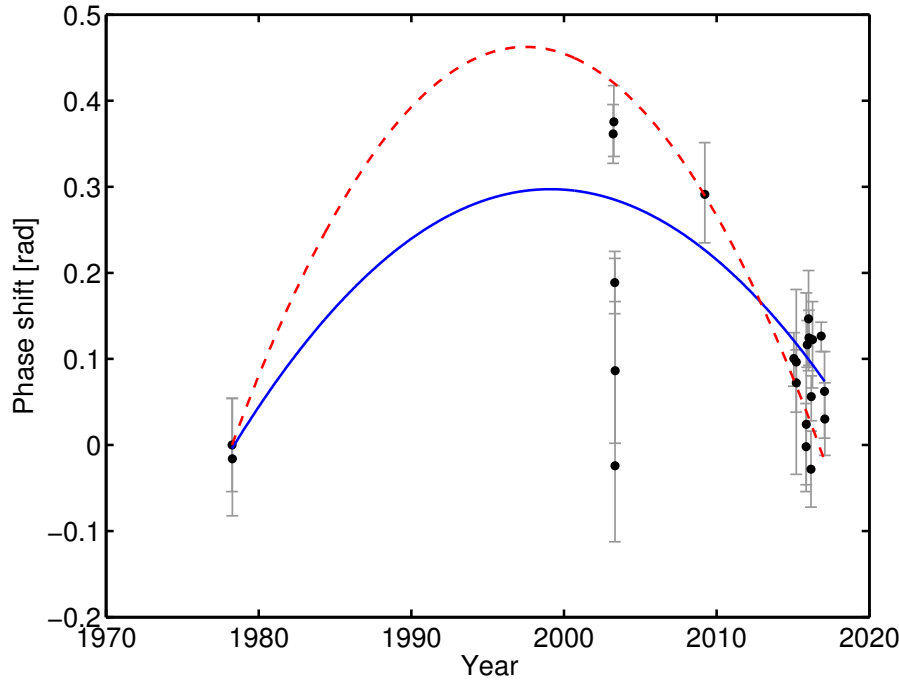

Fig. 7. Phase shift between the best constant-period model of Cacus and the observed lightcurves. Each point in the plot represents a single lightcurve, the error bars represent the uncertainty of the phase shift given the number of points and the level of noise in each lightcurve. The blue curve is the best least-squares quadratic fit to the data taking into account the error bars. The red dashed curve is the quadratic phase shift corresponding to the best YORP model derived with lightcurve inversion.

$(\mathrm{d} a / \mathrm{d} t)_{\mathrm{mod}}$, and the latter with the secular change in the rotation rate $v_{\text {mod }}=(\mathrm{d} \omega / \mathrm{d} t)_{\text {mod }}$. The YORP effect in obliquity is too small to be directly observed and is not reported here. The Yarkovsky $(\mathrm{d} a / \mathrm{d} t)_{\text {mod }}$ value depends on the surface thermal inertia value $\Gamma$, while the YORP $v_{\text {mod }}$ value does not (e.g., Čapek \& Vokrouhlický 2004). We note that our model does not include the YORP component due to the directly reflected 

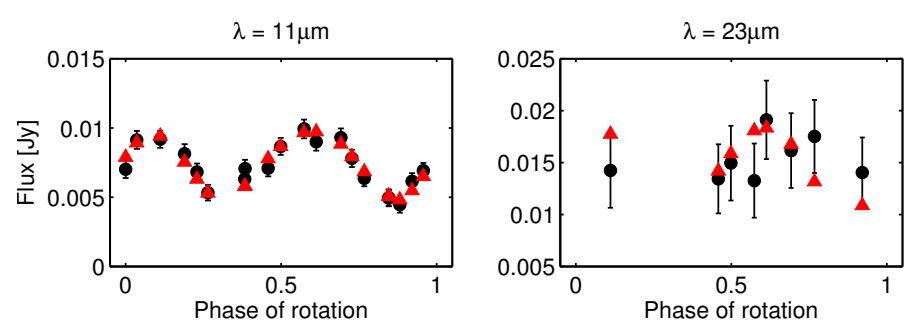

Fig. 8. Comparison between the model (red triangles) and Cacus thermal data observed by WISE on 14 February 2010.

sunlight in optical band. This would require additional unconstrained parameters to be set (e.g., Breiter \& Vokrouhlický 2011). Given the rather small value of the surface albedo, we consider this approximation at the level of neglected effects of surface self-irradiation or lateral thermal communication of the surface facets mentioned above.

Finally, we mention straightforward scaling rules for the size $D$ and bulk density $\rho_{\mathrm{b}}$ (e.g., Vokrouhlický et al. 2015): (i) $(\mathrm{d} a / \mathrm{d} t)_{\text {mod }} \propto 1 /\left(\rho_{\mathrm{b}} D\right)$ and (ii) $v_{\text {mod }} \propto 1 /\left(\rho_{\mathrm{b}} D^{2}\right)$. In what follows we use their nominal values, but our results can be easily recalibrated.

\section{1. (1685) Toro}

We used our nominal values $D=3.5 \mathrm{~km},(\lambda, \beta)=\left(71^{\circ},-69^{\circ}\right)$, and $P=10.19782 \mathrm{~h}$ from Sect. 2.2, and the bulk density of $2.5 \mathrm{~g} \mathrm{~cm}^{-3}$, appropriate for the S-type spectral classification of this body. A sufficiently large range of the thermal inertia values $\Gamma$ was also scanned to see dependence of the Yarkovsky effect on this parameter.

For the YORP effect, we obtained a nominal value $v_{\text {mod }} \simeq$ $10.9 \times 10^{-9} \mathrm{rad} \mathrm{d}^{-2}$. This is somewhat larger, factor $\simeq 3.6$, than the observationally hinted value $v \simeq 3.0 \times 10^{-9} \mathrm{rad} \mathrm{d}^{-2}$, though the latter is quite uncertain in this case. This is the expected level of mismatch due to approximations mentioned above. Nevertheless, there is again a consistency in the possible acceleration of the rotation rate.

Toro has been fortuitously observed by radar on several of its close approaches to the Earth and also has a very long arc of 68 years over which the optical astrometry has been collected. Therefore, in spite of this asteroid's large size, the Yarkovsky effect has been detected fairly well. Our optical data revision, and radar astrometry from January 2016 added to the data set, yield $\mathrm{d} a / \mathrm{d} t=-(1.38 \pm 0.32) \times 10^{-4} \mathrm{au} \mathrm{Myr}^{-1}$. This is in a very good agreement with the predicted $(\mathrm{d} a / \mathrm{d} t)_{\bmod }$ value shown in Fig. 9. The bulk density between 2 and $2.5 \mathrm{~g} \mathrm{~cm}^{-3}$ is the expected value for the $S$-type spectral classification of this body.

\section{2. (161989) Cacus}

We used our nominal values $D=1 \mathrm{~km},(\lambda, \beta)=\left(254^{\circ},-62^{\circ}\right)$, and $P=3.755067 \mathrm{~h}$ from Sect. 2.3. The uncertainties on the pole position and rotation period have a negligible effect on our results. We also set a bulk density $\rho_{\mathrm{b}}=2.5 \mathrm{~g} \mathrm{~cm}^{-3}$, appropriate for the Q-type classification of this body (Thomas et al. 2014; Scheeres et al. 2015), and sampled the surface thermal inertia range from Sect. 2.3. With this set of parameters, we obtained $v_{\text {mod }} \simeq 4.5 \times 10^{-8} \mathrm{rad} \mathrm{d}^{-2}$. While consistently predicting an acceleration of the rotation rate, our theoretical value is about a factor $\simeq 2.4$ higher than the observed value.

We also used optical astrometry of (161989) Cacus available to date and determined $\mathrm{d} a / \mathrm{d} t=-(1.6 \pm 3.3) \times 10^{-4} \mathrm{au} \mathrm{Myr}^{-1}$.

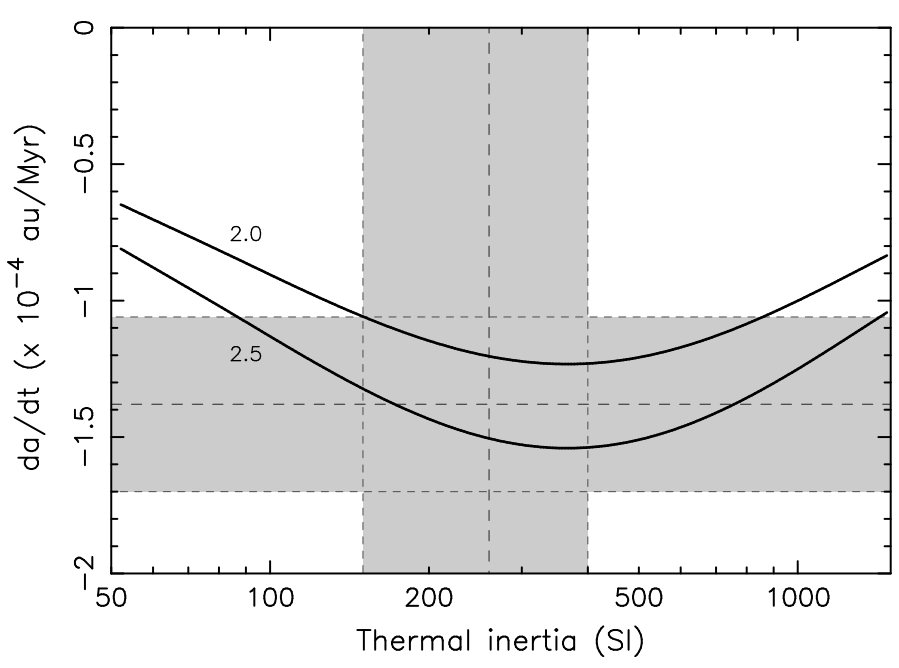

Fig. 9. Computed secular change of the orbital semimajor axis $(\mathrm{d} a / \mathrm{d} t)_{\bmod }$ due to the Yarkovsky effect vs. surface thermal inertia $\Gamma$ for (1685) Toro. Nominal values of the rotation state and size are used. The gray vertical zone indicates the plausible range of $\Gamma$. The horizontal gray area is the Yarkovsky $\mathrm{d} a / \mathrm{d} t$ secular value from the orbit determination ( $1 \sigma$ interval). The Yarkovsky effect has been clearly detected in spite Toro's large size due to radar astrometric observations in four apparitions. The theoretical curves for bulk densities 2 and $2.5 \mathrm{~g} \mathrm{~cm}^{-3}$ closely match the overlap of the observed $\mathrm{d} a / \mathrm{d} t$ and $\Gamma$ values.

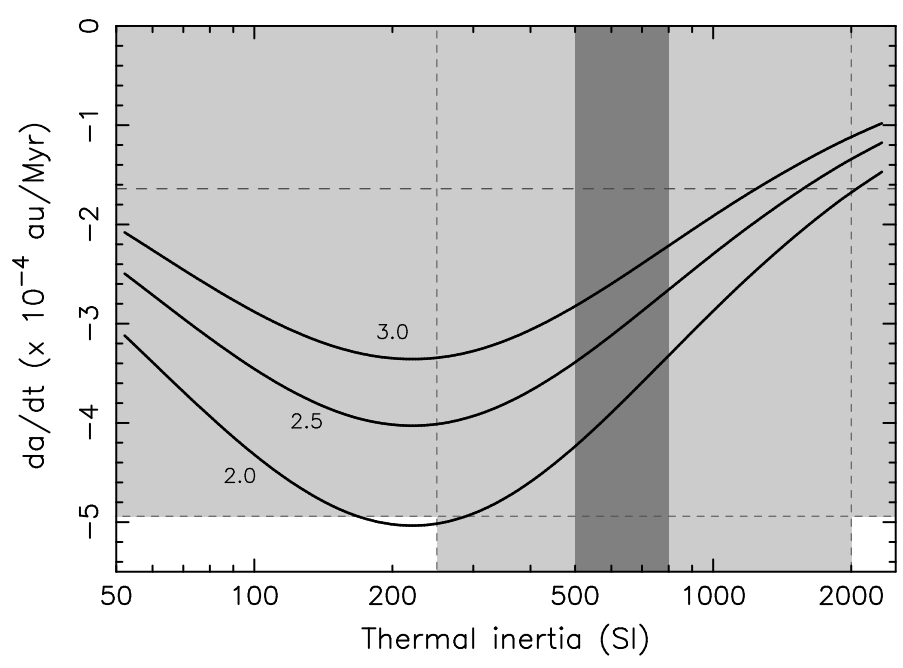

Fig. 10. Same as in Fig. 9, but for asteroid (161989) Cacus. The solid curves show the theoretical dependence for three values of the bulk density $\rho_{\mathrm{b}}=2 \mathrm{~g} \mathrm{~cm}^{-3}, 2.5 \mathrm{~g} \mathrm{~cm}^{-3}$, and $3 \mathrm{~g} \mathrm{~cm}^{-3}$ (see the labels). The gray vertical zone indicates plausible range of $\Gamma$, the dark gray the best-fit values (Sec. 2.3). The horizontal gray area is the Yarkovsky $\mathrm{d} a / \mathrm{d} t \mathrm{sec}-$ ular value from the orbit determination ( $1 \sigma$ interval).

Unfortunately, there are no radar observations of Cacus and the optical data arc is not long enough yet to reveal the Yarkovsky effect in Cacus' orbit. We note that our value supersedes $\mathrm{d} a / \mathrm{d} t=$ (3.35 \pm 2.3$) \times 10^{-4} \mathrm{au} \mathrm{Myr}^{-1}$ from Farnocchia et al. (2013) and $\mathrm{d} a / \mathrm{d} t=(2.6 \pm 2.3) \times 10^{-4} \mathrm{au} \mathrm{Myr}^{-1}$ from Nugent et al. (2012), both of which are compatible with non-detection of the Yarkovsky effect. Figure 10 shows the computed $(\mathrm{d} a / \mathrm{d} t)_{\bmod }$ values as a function of $\Gamma$. Therefore, the current non-detection is very well explained by the small expected $(\mathrm{d} a / \mathrm{d} t)_{\bmod }$ value. 


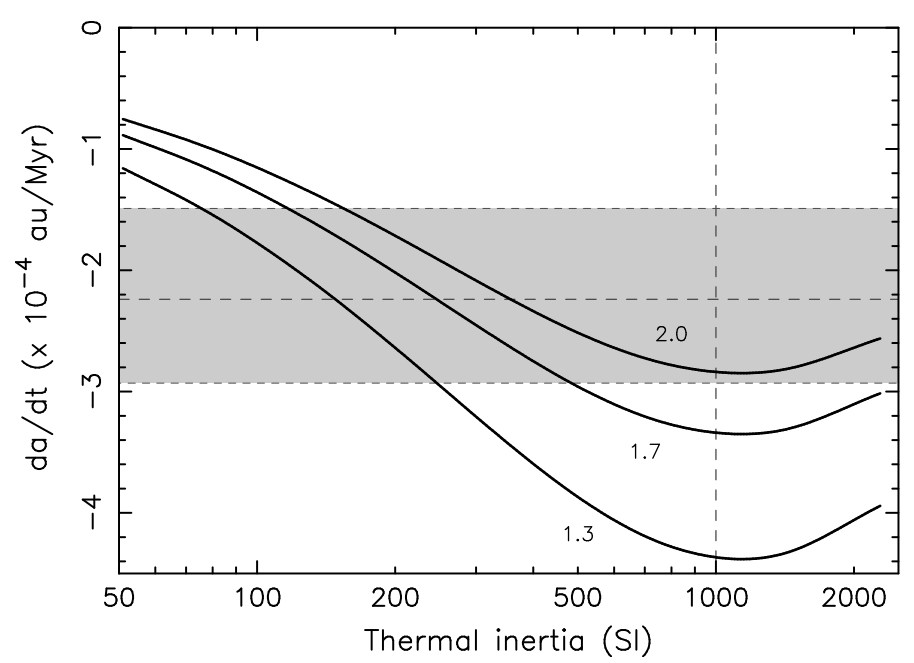

Fig. 11. Same as in Fig. 9, but for asteroid (2100) Ra-Shalom. Here again, the Yarkovsky effect has been fairly well determined thanks to multitude of radar observations in five apparitions. The vertical dashed line indicates the estimated high thermal inertia of Ra-Shalom's surface from Delbó et al. (2003) and Shepard et al. (2008). The three theoretical curves correspond to bulk densities of $1.3,1.7$, and $2 \mathrm{~g} \mathrm{~cm}^{-3}$.

\section{3. (2100) Ra-Shalom}

In this case we used our derived rotation state parameters from Sect. 2.4 and assumed nominal size $D=2.3 \mathrm{~km}$ from Shepard et al. (2008). The spectral classification of this object is somewhat unclear. Binzel et al. (2004) and Bus \& Binzel (2002) classify it as a C- or Xc-type body, but Shepard et al. (2008) give it K-type classification. In this situation, we assume $1.7 \mathrm{~g} \mathrm{~cm}^{-3}$ bulk density, but if higher or lower values turn out to be more appropriate it is necessary to use the recalibration rules mentioned above.

With our nominal values we obtained $v_{\text {mod }} \simeq-10.0 \times$ $10^{-8} \mathrm{rad} \mathrm{d}^{-2}$. Interestingly, the much reduced uncertainty interval for the pole orientation from our solution in Sect. 2.4 (compare with Durech et al. 2012) now allows us to consistently predict the deceleration of the rotation rate for this asteroid. This is intriguing because it would be the first case of this sort. We note, however, that caution should be taken so as not to jump to conclusions. First, the observations only allow us to constrain $v$ to an interval that is compatible with a zero value. Second, our simplified model seems to overpredict the YORP strength by nearly an order of magnitude. This may be partly due to the assumed low density or small size, but also due to missing self-heating effects in our solution. Additionally, the lateral conduction in surface irregularities may be fine-tuned to cancel the negative $v$ value from our model. We conclude that more observations are needed to first set more meaningful constraints on $v$ in this complicated case before proceeding further with theoretical implications.

Unlike the YORP effect, the Yarkovsky effect has been fairly well detected in the orbit of Ra-Shalom. This is again due to very fortuitous circumstance of many radar observations, in this case during five different close approaches to the Earth. Our revision of the data yields $\mathrm{d} a / \mathrm{d} t=-(2.21 \pm 0.72) \times 10^{-4} \mathrm{au} \mathrm{Myr}^{-1}$, somewhat smaller than the value reported by Nugent et al. (2012) and Farnocchia et al. (2013). We suspect the difference is due to new observations and the more recent statistical treatment for optical data we adopted here (Farnocchia et al. 2015; Vereš et al. 2017). Figure 11 shows that the agreement with the predicted value $(\mathrm{d} a / \mathrm{d} t)_{\bmod }$ is fairly good, provided slightly higher than nominal density (and/or larger size) is assumed. This may also help to alleviate the disagreement between the estimated $v_{\text {mod }}$ value and the limits on $v$ from observations.

\section{Conclusion}

In addition to the five asteroids for which YORP has already been detected: (1620) Geographos, (1862) Apollo, (3103) Eger, (25143) Itokawa, and (54509) YORP (see Vokrouhlický et al. 2015, and references therein), we have another clear detection for (161989) Cacus and a hint for YORP in asteroid (1685) Toro. Another recent YORP detection is for asteroid Bennu (Nolan et al., 2017, ACM abstract). A striking feature of these detections is that all the $\mathrm{d} \omega / \mathrm{d} t$ values are positive, which means that for all these asteroids the rotation is accelerated. If there were the same number of asteroids with positive and negative $v$ values, the probability of all seven having the same sign just by chance would be $(1 / 2)^{6} \simeq 1.6 \%$ (or $0.8 \%$ with eight of the same sign, if we include Toro). This low probability might mean that there is an asymmetry between accelerating and decelerating asteroid rotations with a preference for those that spin up. One of the possible mechanisms that would be consistent with this scenario is the transverse heat transport through surface boulders that always leads to acceleration (Golubov \& Krugly 2012) and may have the same order of magnitude as the classical YORP (Ševeček et al. 2015). However, this is still small-number statistics and our sample can be affected by selection bias. It is therefore crucial to significantly enlarge the sample of asteroids with a YORP detection.

A direct observational hint about the asymmetry with which YORP prefers to accelerate the rotation of small asteroids would have other interesting implications. For instance, Pravec et al. (2008; see also updated data in Vokrouhlický et al. 2015) showed that small main-belt and Hungaria asteroids have a rotation-rate distribution that is flat except for an overabundance of slow rotators. This data set may be nicely explained with a YORP-relaxed population, but the significant amount of slowly rotating bodies requires that YORP also decelerates the rotation of asteroids. The relative abundance of slow versus fast rotating bodies in the Pravec et al. (2008) model directly constrains how long asteroids remain in the state of slow rotation before they re-emerge back to regular rotation rates.

Acknowledgements. This work was supported by the Czech Science Foundation (grants GA13-01308S, GA17-00774S, and P209-12-0229). This publication also makes use of data products from NEOWISE, which is a project of the Jet Propulsion Laboratory/California Institute of Technology, funded by the Planetary Science Division of the National Aeronautics and Space Administration. D. Farnocchia conducted this research at the Jet Propulsion Laboratory, California Institute of Technology, under a contract with NASA. The observations at Abastumani were supported by the Shota Rustaveli National Science Foundation, grant FR/379/6-300/14. The Cacus's lightcurve from 2003/02/18.1 was observed in cooperation with R. Michelsen.

\section{References}

Alí-Lagoa, V., Licandro, J., Gil-Hutton, R., et al. 2016, A\&A, 591, A14 Binzel, R. P., Rivkin, A. S., Stuart, J. S., et al. 2004, Icarus, 170, 259 Bottke, Jr., W. F., Vokrouhlický, D., Rubincam, D. P., \& Nesvorný, D. 2006, Annual Review of Earth and Planetary Sciences, 34, 157 Breiter, S., \& Vokrouhlický, D. 2011, MNRAS, 410, 2807 Bus, S. J., \& Binzel, R. P. 2002, Icarus, 158, 146 Čapek, D., \& Vokrouhlický, D. 2004, Icarus, 172, 526 Čapek, D., \& Vokrouhlický, D. 2005, in Dynamics of Populations of Planetary Systems, eds. Z. Knežević, \& A. Milani, IAU Colloq., 197, 171 Carruba, V., Nesvorný, D., \& Vokrouhlický, D. 2016, AJ, 151, 164 Degewij, J., Lebofsky, L., \& Lebofsky, M. 1978, IAU Circ., 3193

Delbó, M., Harris, A. W., Binzel, R. P., Pravec, P., \& Davies, J. K. 2003, Icarus, 166,116

Dunlap, J. L., Gehrels, T., \& Howes, M. L. 1973, AJ, 78, 491 
Ďurech, J., Vokrouhlický, D., Baransky, A. R., et al. 2012, A\&A, 547, A10 Farnocchia, D., Chesley, S. R., Vokrouhlický, D., et al. 2013, Icarus, 224, Farnocchia, D., Chesley, S. R., Chamberlin, A. B., \& Tholen, D. J. 2015, Icarus, 245,94

Golubov, O., \& Krugly, Y. N. 2012, ApJ, 752, L11

Golubov, O., Scheeres, D. J., \& Krugly, Y. N. 2014, ApJ, 794, 22

Greenberg, A. H., Margot, J.-L., Verma, A. K., Taylor, P. A., \& Hodge, S. E. 2017, AJ, in press [arXiv: 1708.05513]

Hanuš, J., Durech, J., Brož, M., et al. 2013, A\&A, 551, A67

Higgins, D. 2008, Minor Planet Bulletin, 35, 30

Higgins, D. 2011, Minor Planet Bulletin, 38, 41

Higgins, D., Pravec, P., Kusnirak, P., et al. 2008, Minor Planet Bulletin, 35, 123

Hoffmann, M., \& Geyer, E. H. 1990, Acta Astron., 40, 389

Kaasalainen, M., Torppa, J., \& Muinonen, K. 2001, Icarus, 153, 37

Kaasalainen, S., Piironen, J., Kaasalainen, M., et al. 2003, Icarus, 161, 34

Koehn, B. W., Bowell, E. G., Skiff, B. A., et al. 2014, Minor Planet Bulletin, 41, 286

Lagerros, J. S. V. 1996, A\&A, 310, 1011

Lagerros, J. S. V. 1997, A\&A, 325, 1226

Lagerros, J. S. V. 1998, A\&A, 332, 1123

Lowry, S. C., Weissman, P. R., Duddy, S. R., et al. 2014, A\&A, 562, A48

Mainzer, A., Bauer, J., Grav, T., et al. 2011, ApJ, 731, 53

Margot, J.-L., Pravec, P., Taylor, P., Carry, B., \& Jacobson, S. 2015, in Asteroids IV, eds. P. Michel, F. E. DeMeo, \& W. F. Bottke (University of Arizona Press), 355

Muinonen, K., Belskaya, I. N., Cellino, A., et al. 2010, Icarus, 209, 542

Nugent, C. R., Margot, J. L., Chesley, S. R., \& Vokrouhlický, D. 2012, AJ, 144, 60

Oey, J. 2011, Minor Planet Bulletin, 38, 221

Ostro, S. J., Campbell, D. B., \& Shapiro, I. I. 1983, AJ, 88, 565
Oszkiewicz, D., Muinonen, K., Bowell, E., et al. 2011, J. Quant. Spectr. Radiat. Trans., 112, 1919

Paolicchi, P., \& Knežević, Z. 2016, Icarus, 274, 314

Pravec, P., Harris, A. W., Vokrouhlický, D., et al. 2008, Icarus, 197, 497

Pravec, P., Vokrouhlický, D., Polishook, D., et al. 2010, Nature, 466, 1085

Pravec, P., Harris, A. W., Kušnirák, P., Galád, A., \& Hornoch, K. 2012a, Icarus, 221,365

Pravec, P., Scheirich, P., Vokrouhlický, D., et al. 2012b, Icarus, 218, 125

Rozitis, B., \& Green, S. F. 2012, MNRAS, 423, 367

Rozitis, B., \& Green, S. F. 2013, MNRAS, 433, 603

Scheeres, D. J., Britt, D., Carry, B., \& Holsapple, K. A. 2015, in Asteroids IV, eds. P. Michel, F. E. DeMeo, \& W. F. Bottke (Tucson: University of Arizona Press), 745

Schuster, H. E., Surdej, A., \& Surdej, J. 1979, A\&AS, 37, 483

Shepard, M. K., Clark, B. E., Nolan, M. C., et al. 2008, Icarus, 193, 20

Thomas, C. A., Emery, J. P., Trilling, D. E., et al. 2014, Icarus, 228, 217

Ševeček, P., Brož, M., Čapek, D., \& Durech, J. 2015, MNRAS, 450, 2104

Ševeček, P., Golubov, O., Scheeres, D. J., \& Krugly, Y. N. 2016, A\&A, 592, A115

Vereš, P., Farnocchia, D., Chesley, S. R., \& Chamberlin, A. B. 2017, Icarus, 296, 139

Vokrouhlický, D., Nesvorný, D., \& Bottke, W. F. 2003, Nature, 425, 147

Vokrouhlický, D., Durech, J., Polishook, D., et al. 2011, AJ, 142, 159

Vokrouhlický, D., Bottke, W. F., Chesley, S. R., Scheeres, D. J., \& Statler, T. S. 2015, in Asteroids IV, eds. P. Michel, F. E. DeMeo, \& W. F. Bottke (Tucson: University of Arizona Press), 509

Vokrouhlický, D., Pravec, P., Ďurech, J., et al. 2017, AJ, 153, 270

Warner, B. D. 2013, Minor Planet Bulletin, 40, 26

Warner, B. D. 2017, Minor Planet Bulletin, 44, 223

Wright, E. L., Eisenhardt, P. R. M., Mainzer, A. K., et al. 2010, AJ, 140, 1868 
J. Ďurech et al.: YORP and Yarkovsky effects in asteroids (1685) Toro, (2100) Ra-Shalom, (3103) Eger, and (161989) Cacus

\section{Appendix A: Additional tables}

Table A.1. Aspect data for new or unpublished observations of (1685) Toro.

\begin{tabular}{|c|c|c|c|c|c|c|}
\hline Date & $\begin{array}{c}r \\
{[\mathrm{AU}]}\end{array}$ & $\begin{array}{c}\Delta \\
{[\mathrm{AU}]}\end{array}$ & $\begin{array}{c}\alpha \\
{[\mathrm{deg}]}\end{array}$ & $\begin{array}{c}\lambda \\
{[\mathrm{deg}]}\end{array}$ & $\begin{array}{c}\beta \\
{[\mathrm{deg}]}\end{array}$ & Obs. \\
\hline 19960716.0 & 1.155 & 0.278 & 54.0 & 358.8 & 20.7 & Sim \\
\hline 19960716.9 & 1.149 & 0.273 & 54.9 & 0.4 & 21.5 & Sim \\
\hline 19960717.0 & 1.148 & 0.272 & 55.0 & 0.6 & 21.5 & Sim \\
\hline 19960720.9 & 1.120 & 0.251 & 59.6 & 8.5 & 25.0 & Sim \\
\hline 20040724.0 & 1.077 & 0.273 & 69.8 & 25.1 & 24.8 & $\mathrm{Kh}$ \\
\hline 20040913.1 & 0.787 & 0.525 & 98.2 & 121.1 & 13.6 & $\mathrm{Kh}$ \\
\hline 20120707.9 & 1.174 & 0.379 & 56.7 & 0.3 & 14.4 & $\mathrm{Kh}$ \\
\hline 20120708.9 & 1.167 & 0.372 & 57.4 & 1.9 & 15.0 & $\mathrm{Kh}$ \\
\hline 20120709.0 & 1.167 & 0.372 & 57.5 & 1.9 & 15.0 & $\mathrm{Kh}$ \\
\hline 20120709.9 & 1.160 & 0.366 & 58.3 & 3.5 & 15.5 & $\mathrm{Kh}$ \\
\hline 20120724.0 & 1.060 & 0.306 & 73.4 & 31.5 & 22.6 & $\mathrm{Si}$ \\
\hline 20120730.0 & 1.018 & 0.302 & 80.9 & 45.9 & 24.4 & $\mathrm{Kh}$ \\
\hline 20120730.9 & 1.011 & 0.302 & 82.1 & 48.2 & 24.6 & $\mathrm{Kh}$ \\
\hline 20120804.0 & 0.983 & 0.308 & 86.9 & 57.9 & 24.8 & $\mathrm{Ab}$ \\
\hline 20130407.2 & 1.809 & 0.839 & 11.6 & 190.6 & -20.2 & Pro \\
\hline 20130408.2 & 1.812 & 0.843 & 11.8 & 190.1 & -20.2 & Pro \\
\hline 20150701.5 & 1.650 & 0.736 & 23.3 & 239.4 & -4.3 & BMO \\
\hline 20150703.5 & 1.640 & 0.741 & 24.8 & 238.6 & -4.0 & $\mathrm{BMO}$ \\
\hline $20150^{\circ}$ & 1.635 & 0.743 & 25.6 & 238.3 & -3.8 & $\mathrm{BMO}$ \\
\hline 20150705.4 & 1.631 & 0.746 & 26.2 & 238.0 & -3.7 & $\mathrm{BMO}$ \\
\hline 20150706.5 & 1.626 & 0.748 & 26.9 & 237.7 & -3.5 & $\mathrm{BMO}$ \\
\hline 20160122.5 & 0.975 & 0.157 & 88.6 & 23.5 & -19.3 & DRO \\
\hline 20160204.6 & 1.066 & 0.200 & 61.2 & 65.9 & -27.4 & DRO \\
\hline 20160205.6 & 1.073 & 0.206 & 59.8 & 68.4 & -27.5 & DRO \\
\hline 20160208.6 & 1.095 & 0.226 & 56.0 & 75.2 & -27.4 & DRO \\
\hline 20160209.6 & 1.102 & 0.233 & 54.9 & 77.2 & -27.3 & DRO \\
\hline 20160210.6 & 1.109 & 0.240 & 53.9 & 79.2 & -27.2 & DRO \\
\hline 20160211.6 & 1.116 & 0.247 & 52.9 & 81.0 & -27.0 & DRO \\
\hline 20160212.6 & 1.123 & 0.255 & 52.0 & 82.8 & -26.8 & DRO \\
\hline 20160213.6 & 1.130 & 0.263 & 51.2 & 84.4 & -26.7 & DRO \\
\hline 20160214.5 & 1.137 & 0.270 & 50.5 & 85.9 & -26.5 & DRO \\
\hline 20160217.6 & 1.159 & 0.295 & 48.4 & 90.3 & -25.8 & DRO \\
\hline 20160222.2 & 1.191 & 0.335 & 46.1 & 95.9 & -24.8 & PDO \\
\hline 20160223.2 & 1.198 & 0.344 & 45.7 & 96.9 & -24.6 & PDO \\
\hline 20160224.2 & 1.205 & 0.353 & 45.4 & 98.0 & -24.3 & PDO \\
\hline 20160224.8 & 1.210 & 0.359 & 45.1 & 98.6 & -24.2 & $\mathrm{Ab}$ \\
\hline 20160225.2 & 1.213 & 0.363 & 45.0 & 99.0 & -24.1 & PDO \\
\hline 20160226.3 & 1.220 & 0.373 & 44.6 & 100.0 & -23.9 & PDO \\
\hline 20160226.8 & 1.224 & 0.378 & 44.5 & 100.5 & -23.7 & $\mathrm{Ab}$ \\
\hline 20160227.7 & 1.230 & 0.387 & 44.2 & 101.4 & -23.5 & $\mathrm{Ab}$ \\
\hline 20160228.2 & 1.233 & 0.391 & 44.1 & 101.8 & -23.4 & PDO \\
\hline 20160229.8 & 1.244 & 0.406 & 43.7 & 103.2 & -23.1 & $\mathrm{Ab}$ \\
\hline 20160316.9 & 1.352 & 0.575 & 41.2 & 114.6 & -19.7 & $\mathrm{BE}$ \\
\hline 20160405.7 & 1.475 & 0.809 & 40.0 & 125.7 & -16.4 & $\mathrm{Si}$ \\
\hline 20160406.7 & 1.481 & 0.821 & 39.9 & 126.2 & -16.3 & $\mathrm{Ab}$ \\
\hline 20160409.8 & 1.498 & 0.860 & 39.8 & 127.8 & -15.8 & $\mathrm{Ab}$ \\
\hline 20160502.8 & 1.620 & 1.162 & 38.2 & 139.1 & -13.1 & $\mathrm{Ab}$ \\
\hline 20160504.7 & 1.630 & 1.188 & 38.0 & 140.0 & -12.9 & $\mathrm{Ab}$ \\
\hline 20160508.8 & 1.649 & 1.243 & 37.7 & 142.0 & -12.5 & $\mathrm{Ab}$ \\
\hline
\end{tabular}

Notes. The table lists Toro's distance from the Sun $r$ and from the Earth $\Delta$, the solar phase angle $\alpha$, the geocentric ecliptic coordinates of the asteroid $(\lambda, \beta)$, and the observatory or source (DK - Danish telescope, La Silla, $1.54 \mathrm{~m}$; BMO - Blue Mountains Observatory, $35 \mathrm{~cm}$, $\mathrm{BE}$ - Blue Eye, Ondřejov, $60 \mathrm{~cm} ; \mathrm{Si}$ - Simeiz Observatory, Crimea, $1 \mathrm{~m} ; \mathrm{Ab}$ - Abastumani Observatory, Georgia, $70 \mathrm{~cm} ; \mathrm{Kh}$ - Astronomical Observatory, Kharkiv, $70 \mathrm{~cm}$; Pro - Prompt Observatory, Cerro Tololo, $41 \mathrm{~cm}$; PDO - Palmer Divide Observatory, $35 \mathrm{~cm}$; DRO - Darling Range Observatory, $30 \mathrm{~cm}$ ).
Table A.2. Aspect data for all available observations of (161989) Cacus.

\begin{tabular}{|c|c|c|c|c|c|c|}
\hline Date & $\begin{array}{c}r \\
{[\mathrm{AU}]}\end{array}$ & $\underset{[\mathrm{AU}]}{\Delta}$ & $\begin{array}{c}\alpha \\
{[\mathrm{deg}]}\end{array}$ & $\begin{array}{c}\lambda \\
{[\mathrm{deg}]}\end{array}$ & $\begin{array}{c}\beta \\
{[\mathrm{deg}]}\end{array}$ & Obs \\
\hline 19780301.2 & 1.131 & 0.142 & 8.4 & 153.5 & -6.6 & $\mathrm{~S} 79$ \\
\hline 19780308.3 & 1.107 & 0.128 & 26.0 & 142.1 & 14.8 & D78 \\
\hline 20030218.1 & 1.186 & 0.232 & 28.5 & 143.5 & -34.5 & DK \\
\hline 20030305.8 & 1.134 & 0.181 & 35.2 & 124.1 & -7.6 & Mod \\
\hline 20030325.9 & 1.063 & 0.230 & 67.3 & 106.9 & 30.0 & Ond \\
\hline 20030401.8 & 1.039 & 0.262 & 74.1 & 103.3 & 38.0 & Ond \\
\hline 20030404.9 & 1.028 & 0.276 & 76.5 & 101.9 & 40.8 & Ond \\
\hline 20090219.4 & 1.121 & 0.238 & 51.0 & 212.5 & 0.6 & K14 \\
\hline 20141221.3 & 1.253 & 0.904 & 51.2 & 186.4 & -18.0 & DK \\
\hline 20150217.3 & 1.067 & 0.466 & 67.6 & 241.7 & 13.5 & DK \\
\hline 20150217.4 & 1.067 & 0.466 & 67.6 & 241.7 & 13.5 & DK \\
\hline 20150220.3 & 1.056 & 0.458 & 68.9 & 245.8 & 16.3 & DK \\
\hline 20151009.3 & 1.300 & 0.808 & 50.2 & 103.6 & -40.8 & DK \\
\hline 20151013.4 & 1.308 & 0.799 & 49.6 & 105.7 & -42.5 & DK \\
\hline 20151105.3 & 1.343 & 0.745 & 46.6 & 115.3 & -51.9 & DK \\
\hline 20151208.3 & 1.363 & 0.656 & 42.5 & 113.2 & -63.5 & DK \\
\hline 20151215.3 & 1.363 & 0.638 & 41.8 & 108.4 & -64.8 & DK \\
\hline 20160204.2 & 1.309 & 0.577 & 44.6 & 75.6 & -45.7 & DK \\
\hline 20160212.0 & 1.294 & 0.585 & 46.5 & 76.2 & -39.8 & DK \\
\hline 20160310.1 & 1.226 & 0.644 & 53.9 & 84.5 & -19.7 & DK \\
\hline 20161006.1 & 1.089 & 0.344 & 66.0 & 289.2 & -23.1 & DK \\
\hline 20161222.1 & 1.310 & 0.952 & 48.4 & 354.6 & -34.8 & DK \\
\hline 20161231.1 & 1.326 & 1.015 & 47.4 & 1.3 & -33.8 & DK \\
\hline
\end{tabular}

Notes. The table lists Cacus's distance from the Sun $r$ and from the Earth $\Delta$, the solar phase angle $\alpha$, the geocentric ecliptic coordinates of the asteroid $(\lambda, \beta)$, and the observatory or source (S79 - Schuster et al. (1979); D78 - Degewij et al. (1978); K14 - Koehn et al. (2014), Ond Ondřejov observatory, $65 \mathrm{~cm}$; DK - Danish telescope, La Silla, $1.54 \mathrm{~m}$; Mod - Modra observatory, $60 \mathrm{~cm}$ ). 
Table A.3. Aspect data for new observations of (2100) Ra-Shalom.

\begin{tabular}{|c|c|c|c|c|c|c|}
\hline Date & $\begin{array}{c}r \\
{[\mathrm{AU}]}\end{array}$ & $\begin{array}{c}\Delta \\
{[\mathrm{AU}]}\end{array}$ & $\begin{array}{c}\alpha \\
{[\mathrm{deg}]}\end{array}$ & $\begin{array}{c}\lambda \\
{[\mathrm{deg}]}\end{array}$ & $\begin{array}{c}\beta \\
{[\mathrm{deg}]}\end{array}$ & Obs. \\
\hline 20130907.1 & 1.153 & 0.402 & 59.2 & 63.4 & -10.7 & Ond \\
\hline 20130908.1 & 1.150 & 0.395 & 59.3 & 64.2 & -11.3 & Ond \\
\hline 20130910.0 & 1.144 & 0.380 & 59.7 & 65.7 & -12.7 & Ond \\
\hline 20130927.1 & 1.078 & 0.274 & 66.8 & 84.1 & -28.8 & Ond \\
\hline 20130928.1 & 1.073 & 0.269 & 67.6 & 85.6 & -30.0 & Ond \\
\hline 20160810.4 & 1.191 & 0.486 & 57.2 & 38.9 & 6.4 & PDO \\
\hline 20160811.4 & 1.192 & 0.479 & 57.0 & 39.2 & 6.1 & PDO \\
\hline 20160812.4 & 1.192 & 0.472 & 56.7 & 39.5 & 5.8 & PDO \\
\hline 20160813.4 & 1.193 & 0.465 & 56.4 & 39.7 & 5.5 & PDO \\
\hline 20160814.4 & 1.194 & 0.458 & 56.2 & 40.0 & 5.2 & PDO \\
\hline 20160815.4 & 1.194 & 0.451 & 55.9 & 40.3 & 4.8 & PDO \\
\hline 20160816.4 & 1.195 & 0.444 & 55.6 & 40.6 & 4.5 & PDO \\
\hline 20160817.4 & 1.195 & 0.437 & 55.3 & 40.8 & 4.2 & PDO \\
\hline 20160819.4 & 1.195 & 0.423 & 54.8 & 41.3 & 3.4 & PDO \\
\hline 20160820.4 & 1.195 & 0.415 & 54.4 & 41.5 & 3.0 & PDO \\
\hline 20160826.0 & 1.194 & 0.375 & 52.6 & 42.7 & 0.6 & Ond \\
\hline 20160828.0 & 1.193 & 0.361 & 51.8 & 43.0 & -0.4 & Ond \\
\hline 20160830.1 & 1.191 & 0.346 & 51.0 & 43.4 & -1.5 & Ond \\
\hline 20160831.0 & 1.190 & 0.339 & 50.6 & 43.5 & -2.0 & Ond \\
\hline 20160903.0 & 1.187 & 0.318 & 49.3 & 43.8 & -3.9 & Ond \\
\hline 20160910.1 & 1.176 & 0.270 & 45.9 & 44.1 & -9.4 & Ond \\
\hline 20160911.7 & 1.172 & 0.259 & 45.1 & 44.0 & -11.0 & $\mathrm{BMO}$ \\
\hline 20160916.7 & 1.161 & 0.228 & 42.5 & 43.4 & -16.5 & $\mathrm{BMO}$ \\
\hline 201609108 & 1.153 & 0.210 & 41.2 & 42.6 & -20.6 & $\mathrm{BMO}$ \\
\hline 20160922.7 & 1.145 & 0.195 & 40.2 & 41.5 & -25.2 & BMO \\
\hline 20160923.7 & 1.141 & 0.190 & 40.0 & 41.0 & -26.9 & BMO \\
\hline 20160925.7 & 1.135 & 0.181 & 39.8 & 39.8 & -30.6 & BMO \\
\hline 20160926.7 & 1.132 & 0.177 & 39.9 & 39.1 & -32.5 & BMO \\
\hline 201600277 & 1.128 & 0.173 & 40.2 & 38.3 & -34.5 & $\mathrm{BMO}$ \\
\hline 20161008.3 & 1.086 & 0.150 & 51.5 & 20.4 & -58.1 & DK \\
\hline 20161009.1 & 1.082 & 0.150 & 52.9 & 18.0 & -59.7 & DK \\
\hline 20161010.2 & 1.077 & 0.150 & 55.0 & 14.0 & -62.0 & DK \\
\hline 20161013.6 & 1.061 & 0.152 & 61.7 & 358.0 & -67.7 & $\mathrm{BMO}$ \\
\hline 20161014.6 & 1.055 & 0.154 & 63.9 & 351.6 & -69.0 & $\mathrm{BMO}$ \\
\hline 20161015.5 & 1.051 & 0.155 & 65.7 & 346.0 & -69.9 & $\mathrm{BMO}$ \\
\hline 20161017.6 & 1.039 & 0.159 & 70.0 & 331.6 & -71.0 & BMO \\
\hline 20161025.7 & 0.993 & 0.183 & 85.1 & 287.8 & -66.8 & $\mathrm{BMO}$ \\
\hline 20161026.6 & 0.987 & 0.186 & 86.8 & 284.7 & -65.8 & BMO \\
\hline
\end{tabular}

Notes. The table lists Ra-Shalom's distance from the Sun $r$ and from the Earth $\Delta$, the solar phase angle $\alpha$, the geocentric ecliptic coordinates of the asteroid $(\lambda, \beta)$, and the observatory or source (Ond - Ondřejov observatory, $65 \mathrm{~cm}$; DK - Danish telescope, La Silla, $1.54 \mathrm{~m}$; BMO Blue Mountains Observatory, $35 \mathrm{~cm}$; PDO - Palmer Divide Observatory, $35 \mathrm{~cm}$ ). 